\title{
MODELO DE DECISÃO EM GRUPO PARA GERENCIAR PERDAS DE ÁGUA
}

\author{
Danielle Costa Morais * \\ Adiel Teixeira de Almeida \\ Programa de Pós-Graduação em Engenharia de \\ Produção (PPGEP) \\ Universidade Federal de Pernambuco (UFPE) \\ Recife - PE \\ dcmorais@ufpe.br \\ aalmeida@ufpe.br \\ * Corresponding author / autor para quem as correspondências devem ser encaminhadas \\ Recebido em 08/2005; aceito em 07/2006 após 3 revisões \\ Received August 2005; accepted July 2006 after 3 revisions
}

\begin{abstract}
Resumo
O planejamento de ações em sistemas de abastecimento, especialmente quanto à redução de perdas e desperdícios de água, normalmente envolve grupos ou instituições com diferentes objetivos, responsabilidades e interesses. Inclui também uma grande variedade de alternativas para minimização do problema, principalmente em termos de custo e desempenho, fazendo com que a escolha se torne uma tarefa bastante complexa, requerendo uma metodologia específica para tratamento dos conflitos. Com o intuito de contribuir para resolução do problema, este artigo apresenta uma abordagem multicritério fundamentada no emprego do Método PROMÉTHÉE, sendo proposto um modelo para tomada de decisão em grupo. Através de um experimento ilustrativo, busca-se investigar o emprego desta abordagem com quatro decisores, representando aspectos financeiro, técnico, ambiental e social. Assim, os responsáveis por este setor terão uma nova postura frente ao processo decisório, em relação à ação a ser desenvolvida, para uma melhor eficiência operacional e manutenção do sistema, administrando perdas.
\end{abstract}

Palavras-chave: perdas d'água; conflitos; decisão multicritério.

\begin{abstract}
The planning of actions in supply systems, especially related to loss reduction and water wastage, usually involves groups or institutions with different objectives, responsibilities and interests. It also includes several alternatives to minimize the problem, mainly in terms of cost and performance, making the selection of the alternatives a complex task, requiring a specific methodology for treatment of conflicts. To contribute solve the problem, this study presents a multicriteria approach based on PROMÉTHÉE method, proposing a model for group decision-making. Through an illustrative experiment, the employment of this approach is investigated with four decision-makers, representing financial, technical, environmental and social aspects. Thus, people responsible for sanitation division will have a different slant of the decision process, in relation to the action to be developed, for better operation and maintenance efficiency, managing losses.
\end{abstract}

Keywords: water losses; conflicts; multicriteria decision. 


\section{Introdução}

A falta de eficiência no gerenciamento de sistemas de abastecimento de água tem se tornado um sério problema na atualidade. Isto limita o desenvolvimento econômico, degrada o meio ambiente e afeta o bem-estar da sociedade. Estudos mais recentes apresentam graves previsões de que a demanda de água irá exceder o abastecimento sustentável em curto prazo (Coêlho, 2001). No futuro, a situação será ainda mais crítica, com o crescimento populacional e o aumento do padrão de vida.

Muitos sistemas de abastecimento de água vêm apresentando deficiências operacionais, seja pelo elevado incremento na demanda nos últimos anos ou pela falta de manutenção adequada. As interrupções no fornecimento de água são comuns, gerando a insatisfação dos seus usuários. Assim, fica evidente a necessidade de modernização do setor de abastecimento de água diante das exigências impostas pelas sociedades urbanas da atualidade, especialmente induzidas pelos processos de democratização e conscientização ecológica. No entanto, reconhece-se que as empresas de saneamento, públicas ou privadas, estão enfrentando novos desafios, buscando atender a uma multiplicidade de aspectos no âmbito da gestão operacional dos sistemas.

Além disso, tomada de decisão para o gerenciamento de recursos naturais é uma atividade bastante complexa, envolvendo vários stakeholders. É necessária a negociação entre esses diferentes grupos ou indivíduos, os quais têm interesses social, econômico e político conflitantes. Faz-se útil então, um método para facilitar a mediação desse processo de negociação para reduzir consideravelmente o esforço investido em resolver estes conflitos e ajudar os atores envolvidos no processo a achar os melhores resultados. A crescente necessidade por novas soluções técnicas e processos de tomada de decisão mais claros têm atraído interesse dos pesquisadores e gerentes.

Neste contexto, com o intuito de contribuir para o tratamento do processo decisório em questão, neste artigo investiga-se uma abordagem fundamentada no emprego do método multicritério PROMÉTHÉE (Preference Ranking Organization Method for Enrichment Evaluation), elaborando-se um modelo baseado no procedimento para apoio a tomada de decisão em grupo proposto por Macharis et al. (1998). O método PROMÉTHÉE se preocupa em ajudar a encontrar a solução mais conveniente em situações onde os decisores previamente identificaram os critérios e as alternativas, ou seja, não se preocupa com a fase de estruturação, mas sim com a fase de avaliação. Assim, o modelo considera algumas possíveis alternativas e as avalia através de critérios pré-estabelecidos, priorizando as mais adequadas, possibilitando à gestão ganhar uma visão geral do negócio e tornar-se apta a tomar decisões multifuncionais, com possíveis estratégias de solução para minimização de perdas no sistema. Com isso, os responsáveis pelo planejamento neste setor disporão de modelo específico de apoio a decisão de forma a integrar os recursos disponibilizados com os objetivos estratégicos das concessionárias.

Sucintamente, este artigo está organizado da seguinte forma: a seção 2 apresenta uma breve descrição do problema das perdas; a seção 3 aborda o método utilizado; a seção 4 descreve um experimento que tem por objetivo investigar a aplicação da abordagem proposta na problemática de priorização das alternativas para redução das perdas em sistemas de abastecimento, apresentando os resultados obtidos; e, finalmente, a seção 5 apresenta as considerações finais. 


\section{O Problema das Perdas}

Os sistemas de abastecimento de água por sua complexidade e características próprias embutem um certo grau de perda da produção, por isto é utópica a idéia de se obter perda zero neste setor. O problema é que as empresas de saneamento estão convivendo com índices elevados de perdas e conseqüentemente de receita, jogando fora água tratada por falta de um gerenciamento adequado.

No Brasil hoje, de cada dez litros de água que saem das estações de tratamento, quatro em média, não produzem recursos para as companhias de saneamento. São as chamadas perdas de faturamento - físicas e não-físicas - que, conforme o Sistema Nacional de Informações sobre Saneamento (SNIS, 2003), representam cerca de R \$2,5 bilhões/ano.

De acordo com Hirner et al. (1999) apud Venturini et al. (2001), a quantidade de água perdida é um importante indicador da eficiência de uma empresa de abastecimento, tanto em termos absolutos num dado momento, como em termos de tendência ao longo dos anos. Volumes de perda de água anuais altos e com tendência para aumentar, servem como indicador de ineficiência no planejamento e construção, bem como demonstram a deficiência em nível de manutenção e de operação do sistema. A redução das perdas de $45 \%$ para $25 \%$ nas empresas de saneamento brasileiras resultaria na disponibilização de recursos da ordem de R \$ 1,0 bilhão/ano (SNIS, 2003).

Segundo Venturini et al. (2001), a falta de planejamento e manutenção adequada, associadas à escassez de recursos financeiros têm tornado deficientes os sistemas de abastecimento de água. Os sistemas com o passar do tempo se deterioram de maneira natural ou acelerada, dando origem a problemas operacionais que provocam a diminuição da qualidade dos serviços prestados e aumento dos custos operacionais, os quais recairão sobre seus consumidores na forma de tarifas. Estes efeitos podem ser sentidos pelas empresas diante dos altos índices de perdas de água no sistema, principalmente associados a alta pressão de trabalho, gerando um grande número de rupturas; crescente aumento das despesas com manutenção do sistema; aumento significativo do consumo energético, uma vez que, manobras operacionais tendem a compensar o funcionamento deficiente do sistema; aumento do número de reclamações.

Por parte dos consumidores reconhecem-se a má qualidade da água fornecida; freqüentes interrupções das vias públicas para execução de reparos nas redes de distribuição ou redes adutoras; abastecimento de água intermitente, pois o sistema não tem capacidade de suprir a demanda; problemas de pressão da rede muito acima ou abaixo dos limites normalizados, causando danos aos usuários.

Deve-se ter em mente que o atendimento aos usuários do sistema só terá bons resultados quando as empresas de saneamento conseguirem minimizar o déficit de água, maximizar a segurança e a confiabilidade do sistema, maximizar a qualidade do serviço prestado, empregar tarifas adequadas na cobrança dos serviços, etc. A qualidade do serviço de abastecimento de água é traduzida não apenas com base na confiabilidade do atendimento à demanda, mas também a outros aspectos importantes, tais como:

- a tarifa cobrada pelo serviço;

- grau de utilização e comprometimento dos recursos hídricos disponíveis em termos quantitativos e qualitativos;

- a sustentabilidade dos recursos e serviços a médio e longo prazo; 
- a garantia do atendimento aos requisitos de pressão mínima e máxima na rede;

- a operação com o mínimo de perdas.

Existem várias alternativas para minimização desse problema, sendo estas largamente conhecidas pela comunidade científica do setor. O problema em questão é a decisão sobre qual alternativa a ser implantada. Como no Brasil as dificuldades encontradas no gerenciamento de sistemas públicos são enormes, torna-se bastante complexa essa avaliação. Assim, analisando a situação em que se encontram os sistemas de abastecimento de água (com $40 \%$ de perdas em média), pode-se concluir que qualquer alternativa que seja implantada trará melhoria ao sistema, no entanto, os recursos para investimentos são escassos e é natural pensar em investir na alternativa de gere o melhor retorno.

Neste contexto, torna-se necessário uma metodologia de apoio a decisão multicritério para ajudar os gerentes desse setor a avaliar as ações potenciais, com relação a critérios préestabelecidos e levando em consideração as características de cada sistema. Assim, o propósito deste trabalho é aplicar o método PROMÉTHÉE, de forma a avaliar as alternativas a serem implantadas sob diferentes aspectos: financeiros, técnicos, ambientais e sociais, considerando o ponto de vista de diferentes decisores. Nessa perspectiva, poderão ser adotadas medidas tanto preventivas, como reparadoras, a fim de que seja obtida uma distribuição de forma justa que atenda plenamente o consumidor, não agrida o meio ambiente e seja coerente com a realidade da empresa concessionária da água (Al-Rashdan et al., 1999). O problema na tomada de decisão é eleger quais componentes adicionar ou utilizar, com o objetivo de maximizar os benefícios resultantes dessas alterações impostas ao sistema, dado que os recursos são insuficientes para propiciar a melhoria exigida no sistema como um todo.

\section{Método Utilizado}

São encontradas algumas dificuldades na tomada de decisão para o gerenciamento de sistemas de abastecimento de água no que diz respeito à escolha ou priorização das alternativas a serem implantadas para a redução de perdas, quando se trata de avaliações de aspectos subjetivos, tais como: impactos sociais, compatibilidade com as metas das concessionárias, influência na melhoria da manutenção e controle operacional. Assim, percebe-se claramente que este é um problema multicritério e deve ser tratado como tal.

$\mathrm{O}$ apoio multicritério a decisão consiste em um conjunto de métodos para auxiliar pessoas e organizações a tomarem decisões, esclarecendo o problema e avaliando as alternativas através de múltiplos critérios, os quais são conflitantes na maioria dos casos (Gomes et al., 2002; Almeida \& Costa, 2003; Gomes et al., 2004). Segundo Vincke (1992), esses modelos e métodos seguem duas principais vertentes: a Escola Americana, a qual consiste na agregação dos diferentes critérios em uma única função de síntese (por exemplo, MAUT, AHP, TOPSIS); e, a Escola Francesa ou Européia, a qual consiste em construir uma relação de sobreclassificação que representa as preferências estabelecidas pelo decisor e explorá-las a fim de ajudar a resolver seu problema (por exemplo, ELECTRE, PROMÉTHÉE).

A escolha do método a ser empregado depende do tipo de problema em análise, do contexto estudado, dos atores envolvidos, da estrutura de preferência e do tipo de resposta que se deseja alcançar, ou seja, qual a problemática de referência (Gomes et al., 2002). Dessa forma, o problema em questão, como já caracterizado anteriormente, visa uma priorização de 
alternativas, sofrendo a influência de vários agentes de decisão por se tratar de investimento que vai refletir em aspectos técnicos, ambientais e sociais. Cada um desses aspectos será considerado como critério na avaliação, e, portanto, requer uma informação intercritério que corresponda à sua importância relativa. Para esses casos, freqüentemente faz-se uso dos métodos da Escola Francesa que utilizam a abordagem outranking (existem traduções diferenciadas para o português, tais como: superação, subordinação, prevalência e sobreclassificação) ou surclassement, da terminologia original em francês (Roy, 1985).

Dentre os métodos baseados na relação de sobreclassificação, decidiu-se então, selecionar o método PROMÉTHÉE II para trabalhar este problema, cuja problemática é a ordenação das alternativas para redução de perdas de água, por sua vantagem em requerer uma informação adicional muito clara, que pode ser facilmente obtida e gerenciada tanto pelo decisor como pelo analista. Esta informação adicional, a noção de critério generalizado, é introduzida com a finalidade de captar a amplitude das diferenças entre as avaliações de cada um dos critérios, enriquecendo a estrutura de preferência. Além do mais, é um método flexível, oferecendo dois graus de liberdade ao decisor: o primeiro é relativo à seleção do tipo de função de preferência e o segundo os limiares a definir (Brans \& Mareschal, 1994).

Um outro aspecto que foi considerado na escolha do PROMÉTHÉE II ao invés de outros métodos da Escola Francesa, como métodos da família ELECTRE, por exemplo, é o fato de que o método ELECTRE exige uma intensa interação entre os decisores e o analista, a fim de tornar clara a parametrização utilizada, além do que esses parâmetros do modelo (pesos, limiares etc.) devem representar o consenso do grupo por unanimidade ou pelo menos de uma grande maioria (Leyva-López \& Fernández-González, 2003). Já com a utilização do método PROMÉTHÉE para tomada de decisão em grupo, os decisores impõem seus critérios individuais, os quais podem ser comuns aos outros decisores ou não, e os avaliam de acordo com seus próprios sistemas de valores, atribuindo pesos, escolhendo as funções de preferência e definindo os limiares, o que proporciona uma maior liberdade ao decisor em expor o seu ponto de vista. Assim, todos os decisores passam a ter uma melhor visão pessoal do problema e a partir daí, é realizada a agregação das preferências, gerando a decisão em grupo. Desse modo, o PROMÉTHÉE II se apresentou mais adequado no contexto da aplicação do modelo, onde são considerados vários agentes decisores, cuja interação apresentava certa dificuldade.

De forma geral o PROMÉTHÉE é de fácil entendimento, de modo que os conceitos e parâmetros envolvidos em sua aplicação têm um significado físico ou econômico de rápida assimilação pelo decisor. Assim, o método propicia a modelagem de preferência de forma simples e de fácil compreensão, usando um paradigma diferente de outras abordagens que agregavam critérios por meio de uma função aditiva. Além disso, os métodos de sobreclassificação não admitem a compensação ilimitada de largas desvantagens e levam em conta o fato que pequenas diferenças entre as avaliações das alternativas nem sempre são significantes (Vincke, 1992).

Este método foi proposto pela primeira vez em 1982 e desde então não deixou de ser objeto de desenvolvimento e adaptações complementares (Brans et al., 1986), tendo sido aplicado com sucesso em vários problemas de diferentes naturezas (Cavalcante \& Almeida, 2005; Almeida \& Costa, 2002; Ülengin et al., 2001; Raju \& Kumar, 1999). As seguintes implementações do PROMÉTHÉE são descritas na literatura (Brans \& Vincke, 1985; Brans et al., 1986; Taleb \& Mareschal, 1995): 
- PROMÉTHÉE I - estabelece uma pré-ordem parcial entre as alternativas, utilizado para problemática de escolha.

- PROMÉTHÉE II - estabelece uma pré-ordem completa entre as alternativas, utilizado para problemática de ordenação.

- PROMÉTHÉE III - ampliação da noção de indiferença, com tratamento probabilístico dos fluxos (preferência intervalar).

- PROMÉTHÉE IV - estabelece uma pré-ordem completa ou parcial, utilizado para problemática de escolha e ordenação destinadas às situações em que o conjunto de soluções viáveis é contínuo.

- PROMÉTHÉE V - nesta implementação, após estabelecer uma ordem completa entre as alternativas, com o PROMETHEE II, são introduzidas restrições, identificadas no problema, para as alternativas selecionadas; incorpora-se uma filosofia de otimização inteira.

- PROMÉTHÉE VI - estabelece uma pré-ordem completa ou parcial, utilizada para problemática de escolha e ordenação. Destinado às situações em que o decisor não consegue estabelecer um valor fixo de peso para cada critério.

- PROMÉTHÉE - GAIA - extensão dos resultados do PROMETHEE, através de um procedimento visual e interativo.

Como citado anteriormente, este modelo utilizará o método PROMÉTHÉE II, que objetiva uma ordenação completa das alternativas evitando qualquer incomparabilidade, característica que melhor se adequou à problemática considerada.

A utilização do PROMÉTHÉE II exige o conhecimento de alguns conceitos utilizados em sua fase de construção da relação de sobreclassificação:

- $w_{j}$ é o peso do critério $\mathrm{j}$, significa a importância que o critério tem em relação aos outros critérios.

- $g_{\mathrm{j}}(\mathrm{a})$ é o valor ou o desempenho da alternativa a no critério j.

- q representa um limite de indiferença, o maior valor para $\left[\mathrm{g}_{\mathrm{j}}(\mathrm{a})-\mathrm{g}_{\mathrm{j}}(\mathrm{b})\right]$ abaixo do qual existe uma indiferença.

- $p$ representa o limite de preferência, o menor valor para $\left[\mathrm{g}_{\mathrm{j}}(\mathrm{a})-\mathrm{g}_{\mathrm{j}}(\mathrm{b})\right]$ acima do qual existe uma preferência estrita.

- $\mathrm{F}_{\mathrm{j}}(\mathrm{a}, \mathrm{b})$ é a função de preferência, valor que varia de 0 a 1 e representa o comportamento ou atitude do decisor frente as diferenças provenientes da comparação par a par entre as alternativas, para um dado critério, indicando a intensidade da preferência da diferença $\mathrm{g}_{\mathrm{j}}(\mathrm{a})-\mathrm{g}_{\mathrm{j}}(\mathrm{b})$ (Brans \& Mareschal, 2002). Comumente, são apresentadas seis formas mais utilizadas, conforme Tabela 1: 
Tabela 1 - Formas das funções de preferência (Adaptado de Brans et al., 1986).

\begin{tabular}{|c|c|c|c|}
\hline \multicolumn{3}{|c|}{$\underline{\text { Critério }}$ (onde d é a diferença na performance $\left(\mathrm{g}_{\mathrm{j}}(a)-\mathrm{g}_{\mathrm{j}}(b)\right.$} & Parâmetros \\
\hline $\begin{array}{c}\text { Critério } \\
\text { usual }\end{array}$ & $H(d)^{\prime}$ & $\begin{array}{l}H(d)=\left\{\begin{array}{l}0 \text { se } d=0 \\
1 \text { se } d \neq 0\end{array}\right. \\
\text { Há uma indiferença entre } a \text { e } b \text { se e } \\
\text { somente se } \mathrm{g}(a)=g(b) \\
\text { Qualquer diferença entre a avaliação das } \\
\text { alternativas, implica em preferência estrita. }\end{array}$ & Nenhum \\
\hline $\begin{array}{l}\text { Quase- } \\
\text { critério }\end{array}$ & $H(d)$ & $\begin{array}{l}H(d)=\left\{\begin{array}{l}0 \text { se }-\mathrm{q} \leq \mathrm{d} \leq \mathrm{q} \\
1 \text { se } d<-q \text { ou } d>q\end{array}\right. \\
\text { Duas alternativas são indiferentes enquanto } \\
\text { a diferença entre as avaliações (d) não } \\
\text { ultrapassar o limiar de indiferença. Acima } \\
\text { desse limiar, a preferência é estrita. }\end{array}$ & Q \\
\hline $\begin{array}{l}\text { III } \\
\text { Critério de } \\
\text { Preferência } \\
\text { Linear }\end{array}$ & & $\begin{array}{l}H(d)= \begin{cases}d / p & \text { se }-p \leq d \leq p \\
1 & \text { se } d<-p \text { ou } d>p\end{cases} \\
\text { A preferência do decisor cresce linear- } \\
\text { mente até que o limiar de preferência (p) } \\
\text { seja atingido. Após esse limiar, a prefe- } \\
\text { rência é estrita. }\end{array}$ & $P$ \\
\hline $\begin{array}{c}\text { Critério } \\
\text { nível }\end{array}$ & & $\begin{array}{l}H(d)=\left\{\begin{array}{lll}0 & \text { se } & |\mathrm{d}| \leq q \\
1 / 2 & \text { se } & \mathrm{q}<|\mathrm{d}| \leq p \\
1 & \text { se } & \mathrm{p}<|\mathrm{d}|\end{array}\right. \\
a \text { e } b \text { são considerados como indiferentes } \\
\text { até que a diferença }(\mathrm{d}) \text { entre } \mathrm{g}_{j}(a) \text { e } \mathrm{g}_{j}(b) \\
\text { não ultrapasse } \mathrm{q}_{\mathrm{j}} ; \text { entre } \mathrm{q}_{\mathrm{j}} \text { e } \mathrm{p}_{\mathrm{j}} \text { o grau de } \\
\text { preferência é fraco e acima de } \mathrm{p}_{\mathrm{j}} \text { a prefe- } \\
\text { rência é estrita. }\end{array}$ & $\mathrm{p}, \mathrm{q}$ \\
\hline $\begin{array}{l}\mathrm{V} \\
\text { Critério de } \\
\text { preferência } \\
\text { linear com } \\
\text { zona de } \\
\text { indiferença }\end{array}$ & & 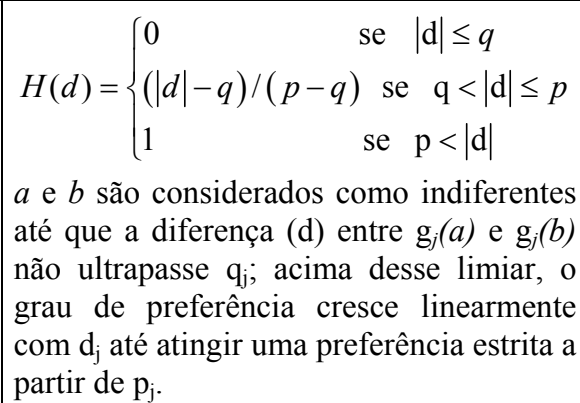 & $\mathrm{p}, \mathrm{q}$ \\
\hline Gaussiana & $\mathrm{H}$ (d) & $\begin{array}{l}H(d)=1-e^{-d^{2} / 2 \sigma^{2}} \\
\text { O desvio padrão }(\sigma) \text { deve ser fixado e a } \\
\text { preferência aumenta segundo uma distri- } \\
\text { buição normal. }\end{array}$ & $\begin{array}{c}\sigma \text { (desvio } \\
\text { padrão) }\end{array}$ \\
\hline
\end{tabular}


- $\Pi(a, b)$ é o grau de sobreclassificação de $a$ em relação a $b$, também chamado de intensidade de preferência multicritério. É calculado por:

$$
\Pi(a, b)=\frac{1}{W} \sum_{j=1}^{n} w_{j} F_{j}(a, b) \quad \text { onde, } W=\sum_{j=1}^{n} w_{j}
$$

- $\Phi^{+}(a)$ é chamado de fluxo de saída e representa a média de todos os graus de sobreclassificação de $a$, com respeito a todas as outras alternativas. É dado pela expressão:

$\Phi^{+}(a)=\sum_{b \in A} \frac{\Pi(a, b)}{n-1}$

Quanto maior $\Phi^{+}(a)$, melhor a alternativa.

- $\Phi^{-}(a)$ é chamado de fluxo de entrada, representa a média de todos os graus de sobreclassificação de todas as outras alternativas sobre $a$. É dado pela expressão:

$$
\Phi^{-}(a)=\sum_{b \in A} \frac{\Pi(b, a)}{n-1}
$$

Quanto menor $\Phi^{-}(a)$, melhor é a alternativa.

- $\Phi(a)$ é chamado de fluxo líquido de sobreclassificação e representa o balanço entre o poder e a fraqueza da alternativa. Quanto maior $\Phi(a)$, melhor a alternativa. É dado pela expressão:

$$
\Phi(a)=\Phi^{+}(a)-\Phi^{-}(a)
$$

\subsection{Procedimento em Grupo}

Problemas de decisão nessa área normalmente envolvem vários decisores. Eles podem representar diferentes departamentos de uma firma, diferentes organizações ou simplesmente diferentes parceiros envolvidos em um projeto. Cada decisor usualmente tem seus objetivos específicos (Cai et al., 2004).

De acordo com Macharis et al. (1998), em decisões em grupo os decisores devem ser reunidos com um facilitador cuja função é conduzir o encontro e moderar discussões diretas entre os decisores e dar assistência ao grupo a fim de obter uma decisão tão eficiente quanto possível.

Critérios individuais ou comuns são considerados. Os critérios comuns são aqueles que são acordados por todos os decisores. Por exemplo, aumento dos benefícios ambientais e redução dos impactos sociais são provavelmente critérios comuns para o problema de gerenciamento de recursos naturais. Critérios individuais podem ser considerados por um ou vários decisores e não necessariamente pelo grupo inteiro, pois, o gerente de uma concessionária de água não terá a mesma avaliação de critérios que um líder comunitário.

Todos os critérios, comuns e individuais, são apresentados numa matriz de avaliação. Esta matriz é apresentada a todos os decisores, os quais fazem seus julgamentos individuais. 
Como a maioria dos métodos de apoio a decisão, o PROMÉTHÉE requer avaliações numéricas. Dessa forma, escalas qualitativas terão que ser transformadas em escalas numéricas.

Uma vez identificadas as alternativas e a avaliação de cada critério, o processo para agregação das informações pode ser iniciado. Para esta finalidade o método PROMÉTHÉE é utilizado. Cada decisor precisa definir os pesos, sendo este a importância relativa entre os critérios, e as funções de preferência para análise de cada critério.

Caso algum decisor considere que determinado critério não seja relevante, este será avaliado com peso igual a zero. Isto significa que tais critérios não serão considerados na análise pessoal desse decisor. Conseqüentemente, embora todos os decisores estejam avaliando a mesma matriz, o número de critérios ativos considerados para cada um pode variar.

De acordo com o procedimento do PROMÉTHÉE, deve ser associada uma função de preferência a cada critério para comparação por pares. Embora cada decisor esteja examinando os mesmos critérios (podendo associar pesos diferentes), a seleção da função de preferência pode ser de forma globalizada ou individual. A globalizada é feita em uma discussão aberta, onde todas as funções de preferência são as mesmas para todos os decisores, e a individual, os decisores analisam o problema separadamente e podem escolher funções de preferência diferentes, cada uma de acordo com o seu respectivo ponto de vista.

Neste contexto, apesar do conjunto de alternativas e de critérios serem idênticos para todos os decisores, as avaliações podem ser completamente diferentes de acordo com as funções de preferência e pesos distribuídos individualmente. Isto depende fortemente do interesse específico dos decisores, que deverão considerar pesos maiores para os critérios que representam sua preocupação no problema, sejam eles técnicos, financeiros, sociais ou ambientais.

Ao final desse estágio, todos os decisores têm uma boa visão pessoal do problema e os valores dos fluxos líquidos obtidos representam as preferências de cada decisor, sendo o maior valor correspondente a melhor alternativa para aquele decisor. Assim, os rankings de cada um são coletados e dispostos numa matriz de avaliação global. Então, dá-se início a um novo problema multicritério com as mesmas alternativas, considerando como critérios os decisores. A Figura 1 ilustra esse procedimento.

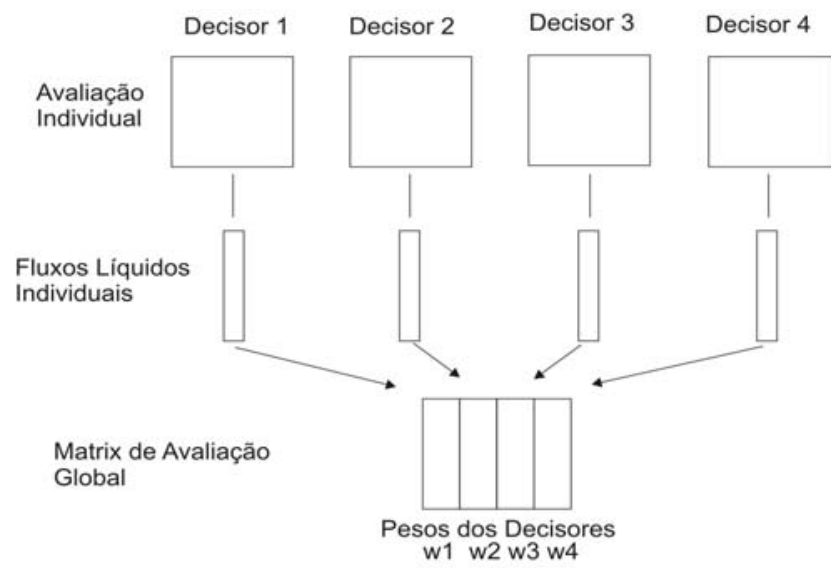

Figura 1 - Evolução da avaliação individual para a global (Adaptado de Macharis et al., 1998). 


\section{Estudo de Caso}

Segundo o SNIS (2003), as companhias estaduais, classificadas como prestadoras de serviços de abrangência regional, são as entidades legalmente constituídas para administrar e operar sistemas, atendendo a vários municípios de forma isolada ou integrada. Por este fato, cada sistema tem suas características próprias e, para aplicar a metodologia de decisão multicritério a fim de priorizar as alternativas de redução de perdas que deverão ser implantadas, é necessário inicialmente diagnosticar a real situação das perdas para o sistema que será analisado.

Com o objetivo de ilustrar a aplicação do modelo, foi elaborado um estudo baseado em um caso real na cidade de Carnaíba, localizada no sertão Pernambucano a $417 \mathrm{~km}$ da capital, com população total de 17.696 habitantes, sendo 6.560 na área urbana e 11.136 na rural (IBGE, 2000). O seu sistema de abastecimento de água é operado pela concessionária estadual, abastece a população urbana (estimada pela companhia em 7.053 habitantes em 2005) e apresenta altos índices de perdas. Esse sistema foi ampliado no ano de 2002, com um alcance de projeto até 2022, ou seja, apresenta um horizonte de tempo de 20 anos. Foram levados em consideração os aspectos característicos da cidade para a avaliação das alternativas em relação aos critérios, sendo realizado um levantamento dos dados referentes a: volumes entregues e micromedidos; informações sobre vida útil dos equipamentos e a quantidade necessária para aferi-los ou substituí-los; informações sobre a quantidade de economias não medidas; informações sobre o nível de automação do sistema; levantamento das pressões na rede a fim identificar a necessidade de setorização, como forma de baixar a pressão na tubulação, diminuindo os vazamentos e estouramentos; dentre outras.

Para caracterizar o problema, deve-se a princípio, identificar onde ocorrem as perdas nos sistemas de abastecimento de água. Vários são os locais possíveis de perdas (captação, adução, elevação, tratamento etc.), sendo na rede de distribuição, onde ocorrem os maiores e mais consideráveis índices de perdas.

\section{- Identificação das alternativas de ação}

Diante da necessidade da cidade analisada através do levantamento dos dados na caracterização do problema, foram propostas as seguintes alternativas para amenizar o problema das perdas de água:

A. substituição ou aferição de hidrômetros;

B. implantação de hidrômetros: para às áreas com estimativa do consumo, geralmente em comunidades de baixa renda. Evita-se o desperdício e perdas não-físicas, pois normalmente o consumo é maior do que o estimado;

C. medição individualizada por apartamento: consiste na instalação de hidrômetros em cada apartamento com vistas ao conhecimento do volume de água consumido por cada um;

D. campanha educativa ao usuário;

E. setorização ou instalação de válvulas redutoras de pressão: inclui a implantação de válvulas e macromedidores nas zonas de pressão;

F. fiscalização contra fraudes;

G. reabilitação de tubulação e conexões;

H. reaproveitamento da água de lavagem das unidades de tratamento. 


\section{- Identificação dos atores}

Em situações como essa, não apenas o representante da concessionária de água da cidade deve ser considerado com decisor, devendo também ser levados em consideração outros stakeholders que influenciam na decisão. Assim, quatro atores foram identificados para fazer parte do processo decisório, representando grupos de interesses específicos, sendo eles:

D1: Concessionária da água (tradicionalmente atua como o órgão responsável pelo sistema);

D2: Engenheiro Projetista (técnico que avalia as questões hidráulicas do sistema);

D3: Agência Ambiental (garantir as condições de sustentabilidade, ações que evitem impactos ao meio ambiente);

D4: Líder Comunitário (representa os interesses dos cidadãos - receber água com boas condições de pressão e evitar aumento das tarifas).

\section{- Identificação e avaliação dos critérios relevantes}

Foram definidos os critérios para a priorização das alternativas de solução:

Cr1 - Custo - custo da implantação da alternativa, sendo considerado que as alternativas de menor valor serão as mais preferíveis.

$\mathrm{Cr} 2$ - Horizonte do investimento - tempo de vida útil da alternativa, considerando o custo de investimento adotado. Esse tempo será considerado em meses, sendo mais preferível aquele que apresentar o maior tempo de vida útil.

Cr3 - Balanço econômico-financeiro - este é um critério mensurável e de expressão pública, que repercute na redução de perdas não físicas. Isto é conseguido com a melhoria da medição real do consumo da população. A redução no custo de produção e distribuição, juntamente com o aumento da arrecadação, terá como conseqüência um aumento significativo no lucro da empresa, tornando-a mais saudável financeiramente.

Cr4 - Redução de desperdício - este critério reflete o potencial da alternativa de reduzir a água gasta irracionalmente, sendo mais preferível nos sistemas com colapso no abastecimento ou subsidiados.

Cr5 - Benefícios ambientais - este critério busca refletir a influência da alternativa no meio ambiente, beneficiando-se aquelas que protegem os mananciais, reduzindo a retirada de água.

Cr6 - Manutenção e operação - este critério visa à diminuição do tempo de vazamento, assim como sua identificação no sistema com maior eficiência e facilidade dos reparos. Também trata a redução das pressões altas na rede, pois são elas as maiores causadoras de vazamentos em canalizações e conexões comprometidas com a vida útil e má estado de conservação.

Cr7 - Aceitabilidade social - este critério visa representar o reflexo da implantação da alternativa dentro das comunidades, sendo valorizada a conscientização da importância do uso racional da água. No sistema em questão, com população atendida $<10.000$ habitantes, esse critério foi avaliado facilmente, porém, para cidades com populações maiores, talvez seja mais adequado fazer a avaliação da aceitação social de forma desagregada, por regiões da cidade, por exemplo.

Os decisores individualmente avaliam os pesos, expressando a importância relativa para cada critério. Cada decisor não necessariamente está interessado em todos os critérios. Assim, será avaliado peso igual a zero para os critérios que determinado decisor julgue não relevante ou não se julgue capaz de avaliar. De acordo com Marcharis et al. (1998) nesta fase da modelagem, cada decisor tem a liberdade de escolher maximizar ou minimizar cada critério, 
além de escolher a sua função de preferência. A Tabela 2 apresenta os pesos, as funções de preferência e respectivos parâmetros avaliados por cada decisor:

Tabela 2 - Pesos, funções de preferência e parâmetros de cada critério, por decisor.

\begin{tabular}{|c|c|c|c|c|c|c|c|c|c|c|c|c|c|}
\hline & & $\mathrm{Cr} 1$ & $\mathrm{Cr} 2$ & \multicolumn{2}{|c|}{$\mathrm{Cr} 3$} & \multicolumn{2}{|c|}{$\mathrm{Cr} 4$} & \multicolumn{2}{|c|}{$\mathrm{Cr} 5$} & \multicolumn{2}{|c|}{ Cr6 } & \multicolumn{2}{|c|}{$\mathrm{Cr} 7$} \\
\hline \multirow{3}{*}{ D1 } & Peso & 0,12 & 0,12 & \multicolumn{2}{|c|}{0,32} & \multicolumn{2}{|c|}{0,04} & \multicolumn{2}{|c|}{0,04} & \multicolumn{2}{|c|}{0,21} & \multicolumn{2}{|c|}{0,15} \\
\hline & Tipo & \begin{tabular}{l|l}
5 & $\min$ \\
\end{tabular} & \begin{tabular}{l|l|}
2 & $\max$ \\
\end{tabular} & 1 & $\max$ & 3 & $\max$ & 3 & $\max$ & 4 & $\max$ & 2 & $\max$ \\
\hline & Parâmetros & $\begin{array}{c}q=5.000 \\
p=50.000\end{array}$ & $q=84$ & \multicolumn{2}{|r|}{ 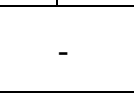 } & \multicolumn{2}{|c|}{$\mathrm{p}=2,00$} & \multicolumn{2}{|c|}{$\mathrm{p}=0,75$} & \multicolumn{2}{|c|}{$\begin{array}{l}q=0,25 \\
p=0,50\end{array}$} & \multicolumn{2}{|c|}{$\mathrm{q}=0,25$} \\
\hline \multirow{3}{*}{ D2 } & Peso & 0,12 & 0,12 & \multicolumn{2}{|c|}{0,24} & \multicolumn{2}{|c|}{0,04} & \multicolumn{2}{|c|}{0,12} & \multicolumn{2}{|c|}{0,32} & \multicolumn{2}{|c|}{0,04} \\
\hline & Tipo & \begin{tabular}{l|l}
3 & $\min$ \\
\end{tabular} & \begin{tabular}{l|l}
2 & $\max$ \\
\end{tabular} & 1 & $\max$ & 3 & $\max$ & 3 & $\max$ & 4 & $\max$ & 2 & $\max$ \\
\hline & Parâmetros & $\mathrm{p}=50.000$ & $q=84$ & & - & \multicolumn{2}{|c|}{$\mathrm{p}=0,50$} & \multicolumn{2}{|c|}{$\mathrm{p}=1$} & \multicolumn{2}{|c|}{$\begin{array}{l}\mathrm{q}=0,25 \\
\mathrm{p}=0,75\end{array}$} & &, 25 \\
\hline & Peso & & & & & & 30 & & 40 & & 15 & & 15 \\
\hline D3 & \begin{tabular}{|l|} 
Tipo \\
\end{tabular} & 0 & 0 & & 0 & 3 & $\max$ & 3 & $\max$ & 1 & $\max$ & 3 & $\max$ \\
\hline & Parâmetros & & & & & & 0,75 & &, 50 & & - & &, 25 \\
\hline & Peso & & & & & & 25 & & 15 & & & & 60 \\
\hline D4 & Tipo & 0 & 0 & & 0 & 3 & $\max$ & 3 & $\max$ & & 0 & 1 & $\max$ \\
\hline & Parâmetros & & & & & & 0,75 & &, 00 & & & & 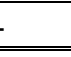 \\
\hline
\end{tabular}

\section{- Julgamento das alternativas em relação aos critérios}

As avaliações de critérios, tanto em valor absoluto como em importância relativa, merecem uma atenção especial, por se tratar do instrumento pelo qual são gerados os dados para o modelo e onde as alternativas manifestam suas características, representando aspectos intrínsecos de cada sistema.

O critério "custo" e "horizonte do investimento" possibilita uma comparação bastante objetiva, sendo o primeiro considerado seu valor por quantias monetárias para implantação da alternativa e o segundo, o tempo de vida útil em meses da alternativa, considerando-se o custo do investimento que foi adotado. Vale salientar que sua mensuração é um pouco complicada, pois exige estudos específicos de cada alternativa para o determinado sistema.

Os outros critérios possuem natureza bastante subjetiva, não possuindo uma escala clara de valores que possa ser utilizada como recurso de comparação. Como nesses critérios, as diferenças entre uma ou outra alternativa podem ser pequenas e de difícil mensuração, adotou-se uma escala de comparação verbal, a qual permite aliar simplicidade da análise a uma maior garantia de objetividade. Os valores atribuídos a cada conceito verbal são apresentados na Tabela 3:

Tabela 3 - Escala para julgamento da importância dos critérios.

\begin{tabular}{|l|c|}
\hline Escala Verbal & Escala Numérica \\
\hline Muito Bom & 1,00 \\
Bom & 0,75 \\
Regular & 0,50 \\
Fraco & 0,25 \\
Desprezível & 0,00 \\
\hline
\end{tabular}


Assim, nesta fase cada decisor faz a sua avaliação individual das alternativas em relação aos critérios que julga relevante. Utilizando o Decision Lab, software baseado no método PROMÉTHÉE e no Plano GAIA (Geometrical Analysis for Interactive Assistance) desenvolvido pelos Professores J. P. Brans e B. Mareschal, sob um acordo exclusivo com a empresa Visual Decision (Geldermann \& Zhang, 2001), é possível analisar o problema sob a perspectiva individual de cada decisor e permite fazer uma análise de sensibilidade, onde as alternativas são projetadas em um plano bidimensional.

Segundo Mareschal \& Brans (1988) o plano GAIA consiste de um módulo de interação visual complementar ao PROMÉTHÉE, fornecendo uma informação gráfica clara sobre o caráter conflitante do critério, neste caso dos decisores, e sobre o impacto dos pesos na decisão final.

A Figura 2 ilustra a visualização do Plano GAIA e os rankings resultantes das análises individuais de cada decisor.

D1
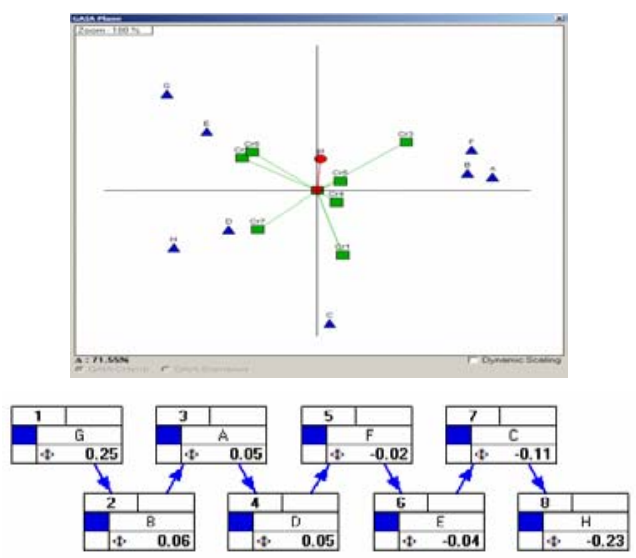

D3
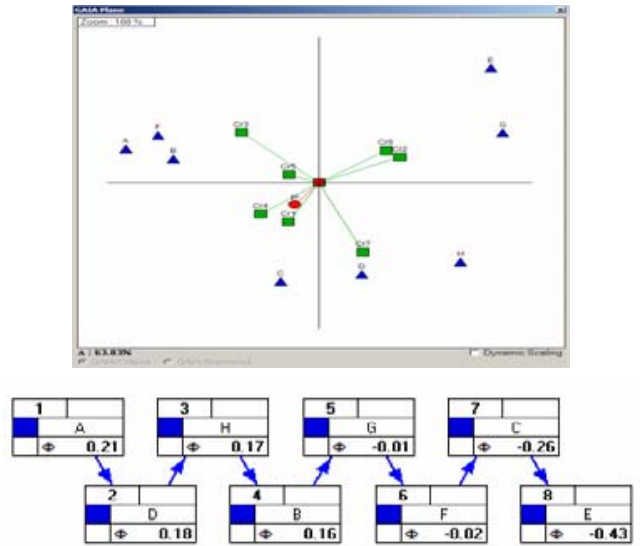

D2

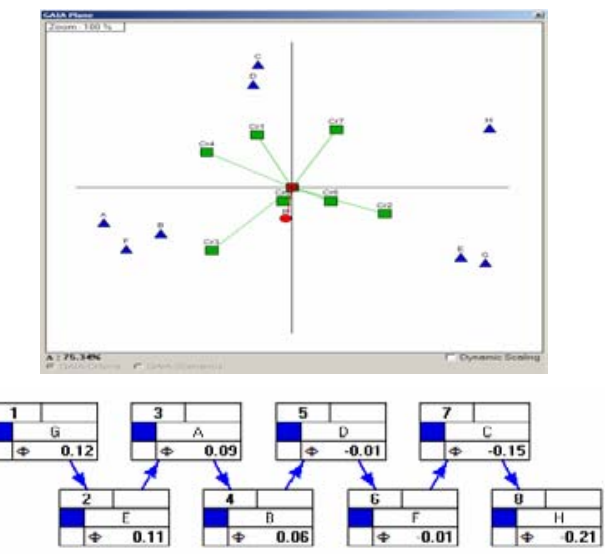

D4

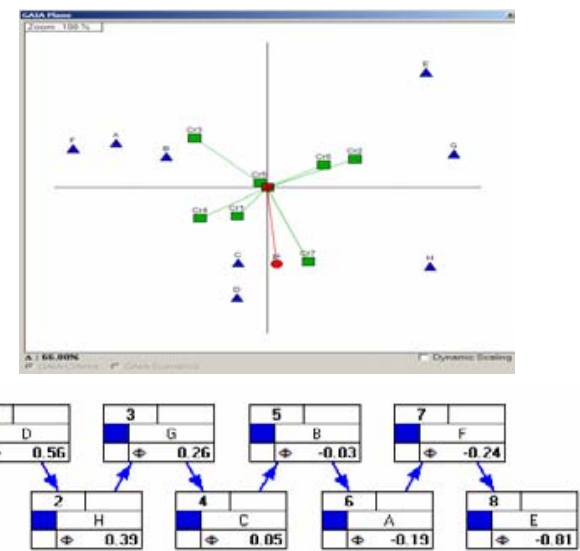

Figura 2 - Visualização do plano GAIA e ranking das alternativas com relação aos pontos de vista dos decisores D1: Concessionária da Água, D2: Engenheiro Projetista, D3: Agência Ambiental e D4: Líder Comunitário. 
Como já era esperado, as alternativas a serem priorizadas não são as mesmas de acordo com as preferências dos diferentes decisores. Os gráficos separadamente oferecem muita informação para serem assimiladas da forma que estão e ao mesmo tempo. Assim, com base nos resultados dos fluxos líquidos individuais, passa-se para a fase de agregação dos resultados, ou avaliação global. Essa fase é a resolução de um novo problema multicritério com o mesmo conjunto de alternativas, mas agora os critérios são os decisores.

\section{- Agregação dos resultados}

De acordo com o procedimento do PROMÉTHÉE, uma função de preferência deve ser avaliada para cada um dos critérios. Entretanto, segundo Macharis et al. (1998), não é realística a avaliação de diferentes funções de preferência para cada um dos decisores. Os fluxos líquidos de cada decisor já são computados com base nas preferências individuais e são expressos na mesma unidade, então, estes valores podem ser diretamente agregados. Consequentemente, é possível fazer o simples cálculo da soma ponderada dos fluxos líquidos individuais, de forma que o fluxo global de uma alternativa para o grupo inteiro será definida assim:

$$
\begin{aligned}
& \Phi^{G}\left(a_{i}\right)=\sum_{r=1}^{R} \phi^{r}\left(a_{i}\right) \omega_{r} \quad \text { sendo } \quad \mathrm{R} \text { decisores, } \mathrm{r}=1,2, \ldots, \mathrm{R}, \text { onde: } \\
& \phi^{r}\left(a_{i}\right) \text { é o fluxo líquido da alternativa } a_{i} \text { para o decisor } \mathrm{r} \\
& \omega_{r} \text { é o peso do decisor sendo } \sum_{\mathrm{r}=1}^{\mathrm{R}} \omega_{r}=1
\end{aligned}
$$

Este fluxo líquido global imediatamente ordena as alternativas de acordo com a preferência global do grupo. Segundo Macharis et al. (1998), este tratamento é chamado de "0-option".

No entanto, para ser utilizado o software Decision Lab no cálculo da avaliação geral, Brans (2005) indica a adoção da função de preferência para os decisores do tipo III (preferência linear) com o limiar de preferência (p) igual a 1. Isto se dá porque a diferença de desempenho das alternativas, $\mathrm{d}=\mathrm{g}_{j}(a)-\mathrm{g}_{j}(b)$, neste caso são os fluxos líquidos resultantes das avaliações individuais dos decisores, portanto são expressos na mesma unidade, variando de -1 a 1 . A Figura 3 ilustra esse comportamento, onde $H(d)$ representa o valor relacionado a função de preferência.

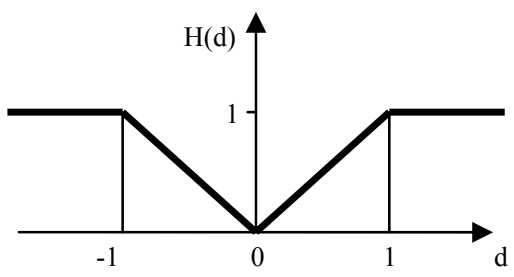

Figura 3 - Critério de preferência linear com o limiar de preferência $p=1$.

É importante destacar que, de acordo com Brans (2005), é comum em decisões em grupo que todos os decisores tenham a mesma importância relativa (pesos iguais), mas neste caso, será considerado um peso maior para o decisor responsável pela Concessionária de Água $(0,40)$, 
enquanto os demais terão pesos iguais $(0,20)$. Garante-se com isso, atenção especial ao ponto de vista dos interesses da companhia, na medida em que será dela as obrigações de implantação, operação e manutenção do sistema. A Tabela 4 apresenta os fluxos líquidos obtidos de cada decisor para a avaliação global.

Tabela 4 - Avaliação global.

\begin{tabular}{|c|c|c|c|c|}
\hline & D1 & D2 & D3 & D4 \\
\hline A & 0,0501 & 0,0929 & 0,2071 & $-0,1929$ \\
\hline B & 0,0626 & 0,0595 & 0,1571 & $-0,0310$ \\
\hline C & $-0,1147$ & $-0,1453$ & $-0,2571$ & 0,0548 \\
\hline D & 0,0484 & $-0,0094$ & 0,1786 & 0,5643 \\
\hline E & $-0,0387$ & 0,1100 & $-0,4286$ & $-0,8095$ \\
\hline F & $-0,0226$ & $-0,0103$ & $-0,0214$ & $-0,2357$ \\
\hline G & 0,2474 & 0,1156 & $-0,0071$ & 0,2619 \\
\hline H & $-0,2325$ & $-0,2129$ & 0,1714 & 0,3881 \\
\hline
\end{tabular}

Com base nesses dados, o PROMÉTHÉE II faz a ordenação das alternativas. A Figura 4 ilustra o resultado dessa ordenação.

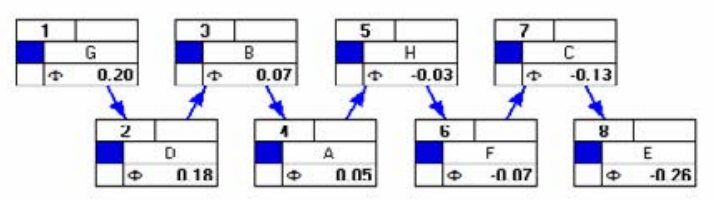

Figura 4 - Resultado da ordenação das alternativas da avaliação global.

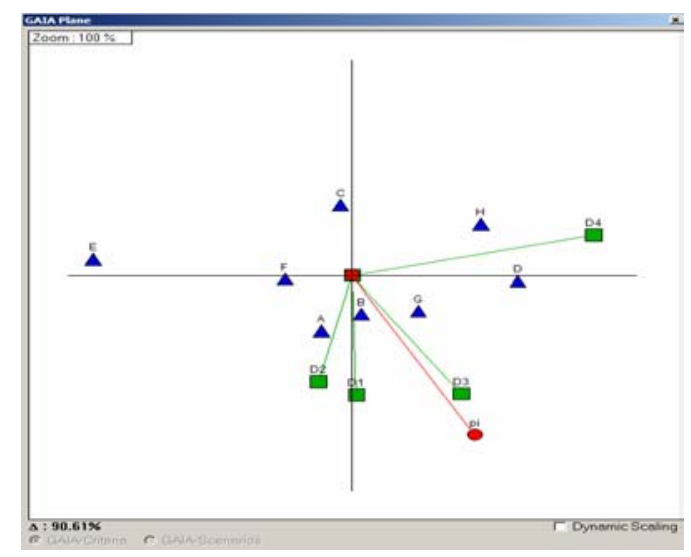

Figura 5 - Visualização do plano GAIA da avaliação global. 
Diante do plano apresentado na Figura 5, algumas características podem ser verificadas de acordo com as propriedades do plano, o qual apresenta um índice de informação preservada de 90\%. Percebe-se que os decisores D1 (concessionária de água) e D2 (engenheiro projetista) estão próximos e aproximadamente orientados na mesma direção, o que representa uma similaridade nas preferências.

Este fato pode ser também claramente observado nos resultados individuais, onde ambos tiveram como resultado para priorização a alternativa G. Desse modo, levando em consideração que o D1 tinha uma maior importância na avaliação global, esta alternativa permaneceu como prioritária na avaliação global.

Um outro aspecto que deve ser ressaltado é que os decisores D1, D2 e D3 estão posicionados aproximadamente de forma ortogonal com o decisor D4, o que caracteriza a independência entre esses decisores.

De uma forma geral, a modelagem do problema através de múltiplos critérios, proveu uma linguagem comum entre os diferentes decisores com pontos de vistas opostos ou conflitantes.

\section{- Análise de sensibilidade}

A análise de sensibilidade foi realizada para verificar a consistência do modelo e para observar o seu comportamento sob algumas variações impostas. Esta análise revela que a negociação alcançada pelos decisores é bastante robusta com relação aos pesos estabelecidos. A simulação foi realizada com uma variação de $\pm 15 \%$ no peso do decisor D1 e o eixo de decisão permaneceu orientado para as alternativas G e D.

Foi feita a análise também com pesos iguais para todos os decisores e a ordenação só apresentou diferença entre a primeira e segunda colocação (sendo nessa análise $\mathrm{D}$ e $\mathrm{G}$, respectivamente primeira e segunda), e as demais alternativas permaneceram com as mesmas colocações. È importante ressaltar que a variação no fluxo líquido entre elas foi pouco significativa.

\section{Considerações Finais}

Neste trabalho foi apresentado um modelo dentro da abordagem multicritério fundamentada no emprego do método PROMÉTHÉE II, com vista ao procedimento de decisão em grupo, com o objetivo de contribuir para o tratamento do problema da priorização de alternativas para redução de perdas e desperdícios de água em sistemas de abastecimento.

Neste aspecto é relevante ressaltar que o modelo foi desenvolvido com a finalidade principal de ilustrar o emprego da abordagem proposta, limitando-se à priorização das alternativas estudadas. É válido ressaltar que possivelmente outros critérios poderiam ter sido considerados na análise, como também outras alternativas de solução incorporadas, o que obviamente modificaria o resultado final da análise. Esta etapa da modelagem do problema pode apresentar variações de acordo com as características do sistema, tais como topografia da cidade, índices de atendimento e intermitência, níveis de pressão na rede, dentre outros de interesse dos decisores.

Assim, o problema de priorização dessas alternativas apresenta-se bastante complexo pela falta de política de ação para empreendimentos dessa natureza, pela escassez de recursos disponíveis neste setor, pela influência de vários grupos de interesses específicos e pela 
subjetividade na análise de alguns aspectos. Dessa forma, o modelo desenvolvido aponta as alternativas de redução de perdas mais favoráveis para cada diferente sistema de abastecimento de água, de forma a não eliminar a subjetividade inerente aos problemas decisórios, apenas buscando tratá-las através do emprego de um método que possibilita a verificação da consistência dos julgamentos.

É preciso sempre ter em mente que o conhecimento pleno das características da rede (cadastro físico e operacional) em conjunto com a utilização de modelos não apenas econômicos (relação custo/benefício), como também multicritério, os quais levam em consideração aspectos hidráulicos, ambientais, sociais e outros que desejar, irá permitir um gerenciamento completo do controle de perdas e desperdício de água por parte da entidade responsável pela distribuição à população, demonstrando uma maior clareza nas decisões tomadas e garantindo a credibilidade dos clientes.

\section{Agradecimentos}

Os autores agradecem ao CNPq e à CAPES pelo apoio financeiro deste estudo.

\section{Referências Bibliográficas}

(1) Almeida, A.T. \& Costa, A.P.C.S. (2003). Aplicações com Métodos Multicritério de Apoio a Decisão. Editora Universitária, Recife.

(2) Almeida, A.T. \& Costa, A.P.C.S. (2002). Modelo de Decisão Multicritério para Priorização de Sistemas de Informação com base no Método PROMÉTHÉE. Gestão \& Produção, 9(2), 201-214.

(3) Al-Rashdan, D.; Al-Kloub, B.; Dean, A. \& Al-Shermmeri, T. (1999). Environmental Impact Assessment and Ranking the Environmental Projects in Jordan. European Journal of Operational Research, 118, 30-45.

(4) Brans, J.P. (2005). Reuniões com o Prof. Jean-Pierre Brans durante o REDs - Recife Decision Support School, I Escola de Apoio a Decisão do Recife, realizado na UFPE no período de 13 a 17 de junho de 2005. Recife.

(5) Brans, J.P. \& Mareschal, B. (2002). PROMÉTHÉE-GAIA: une méthodologie d'aide à la décision en présence de critères multiples. Éditions de L'Université de Bruxelles, Bruxelles.

(6) Brans, J.P. \& Mareschal, B. (1994). PROMCALC \& GAIA: A new decision support system for multicriteria decision aid. Decision Support Systems, 12, 297-310.

(7) Brans, J.P.; Vincke, P. \& Mareschal, B. (1986). How to select and how to rank projects: The PROMETHEE method. European Journal of Operational Research, 24, 228-238.

(8) Brans, J.P. \& Vincke, P.H. (1985). A preference ranking organization method, the PROMETHEE method for MCDM. Management Science, 31(6), 647-656.

(9) Cai, X; Lasdon, L. \& Michelsen, A.M (2004). Group Decision Making in Water Resources Planning Using Multiple Objective Analysis. Journal of Water Resources Planning and Management, 130(1), 4-14. 
(10) Coêlho, A.C. (2001). Manual de Economia de Água (Conservação de Água). Comunigraf Editora, Recife.

(11) Cavalcante, C.A.V. \& Almeida, A.T. (2005). Modelo Multicritério de Apoio a Decisão para o Planejamento de Manutenção Preventiva Utilizando PROMETHEE II em Situações de Incerteza. Pesquisa Operacional, 25(2), 279-296.

(12) Geldermann, J. \& Zhang, K. (2001). Software Review: "Decision Lab 2000". Journal Multi-Criteria Decision Analysis, 10, 317-323.

(13) Gomes, L.F.A.M.; Gomes, C.F.S. \& Almeida, A.T. (2002). Tomada de decisão gerencial: Enfoque Multicritério. Editora Atlas, São Paulo.

(14) Gomes, L.F.A.M.; Araya, M.C.G.; Carignano, C. (2004). Tomada de decisões em cenários complexos: introdução aos métodos discretos do apoio multicritério a decisão. Editora Pioneira Thompson Learning, São Paulo.

(15) IBGE (2000). Instituto Brasileiro de Geografia e Estatística. Censo Demográfico 2000 Resultados do Universo.

(16) Leyva-López, J.C. \& Fernández-González, E. (2003). A new method for group decision support based on ELECTRE III methodology. European Journal of Operational Research, 148(1), 14-27.

(17) Macharis, C.; Brans, J.P. \& Mareschal, B. (1998). The GDSS PROMETHEE Procedure. Journal of Decision Systems, 7, 283-307.

(18) Mareschal, B. \& Brans, J.P. (1988). Geometrical representation for MCDM, the Gaia procedure. European Journal of Operational Research, 34, 69-77.

(19) Raju, K.S. \& Kumar, D.N. (1999) Multicriterion decision making in irrigation planning. Agricultural Systems, 62(2), 117-129.

(20) Roy, B. (1985). Méthodologie Multicritère d'Aide à la Décision. Economica, Paris.

(21) SNIS (2003). Sistema Nacional de Informações sobre Saneamento. Diagnóstico dos Serviços de Água e Esgoto 2003. Disponível em < http://www.snis.gov.br>. Acessado em 29 de mar. 2005.

(22) Taleb, M.F.A. \& Mareschal, B. (1995). Water resources planning in the middle east: application of the PROMETHEE V multicriteria method. European Journal of Operational Research, 81, 500-511.

(23) Ülengin, F.; Topcu, Y.I \& Sahin, S.Ö. (2001). An integrated decision aid system for Bosphorus water-crossing problem. European Journal of Operational Research, 134, 179-192.

(24) Venturini, M.A.A.G; Barbosa, P.S.F. \& Luvizotto Jr., E. (2001). Estudo de Alternativas de Reabilitação para Sistemas de Abastecimento de Água. In: XIV Simpósio Brasileiro de Recursos Hidricos, Aracaju-SE, Brasil.

(25) Vincke, P. (1992). Multicriteria Decision-Aid. Wiley, Bruxelles. 